San Jose State University

SJSU ScholarWorks

Faculty Publications

Valley Foundation School of Nursing

$1-1-2011$

\title{
Changes in temperature of heel skin under pressure in hip surgery patients
}

Vivian K. Wong

San Jose State University, vivian.wong@sjsu.edu

N. A. Stotts

University of California, San Francisco

H. W. Hopf

University of Utah, Salt Lake City

G. A. Dowling

University of California, San Francisco

E. S. Froelicher

University of California, San Francisco

Follow this and additional works at: https://scholarworks.sjsu.edu/nursing_pub

Part of the Nursing Commons

\section{Recommended Citation}

Vivian K. Wong, N. A. Stotts, H. W. Hopf, G. A. Dowling, and E. S. Froelicher. "Changes in temperature of heel skin under pressure in hip surgery patients" Advances in Skin \& Wound Care (2011): 562-570.

https://doi.org/10.1097/01.ASW.0000408466.88880.f8

This Article is brought to you for free and open access by the Valley Foundation School of Nursing at SJSU

ScholarWorks. It has been accepted for inclusion in Faculty Publications by an authorized administrator of SJSU

ScholarWorks. For more information, please contact scholarworks@sjsu.edu. 
Changes in temperature of heel skin under pressure in hip surgery patients

Vivian K. Wong, RN, PhD, Nancy A. Stotts, RN, EdD, FAAN, Harriet W. Hopf, MD,

Glenna A. Dowling, RN, PhD, FAAN, Erika Sivarajan Froelicher, RN, PhD, FAAN

Work should be attributed to:

School of Nursing, University of California, San Francisco

\section{Corresponding author:}

Vivian Wong, RN, PhD

Associate Professor

School of Nursing, San Jose State University

One Washington Square,

San Jose, CA 95192-0057

Tel: (408) 924-3155

Fax: (408) 924-3135

E-mail: Vivian.wong@sjsu.edu

Nancy A. Stotts, RN, EdD, FAAN

Professor, Physiological Nursing, University of California San Francisco,

San Francisco, California

Harriet W. Hopf, MD

Professor, Department of Anesthesiology, University of Utah

Salt Lake City, Utah

Glenna A. Dowling, RN, PhD, FAAN

Professor, Physiological Nursing, University of California San Francisco, San Francisco, California

Erika S. Froelicher, RN, PhD, FAAN

Professor, Physiological Nursing, School of Nursing, and

Department of Epidemiology, School of Medicine

University of California San Francisco,

San Francisco, California 


\section{Funding:}

This study was funded by the Individual National Research Service Award NR008317-03, the 2004 American Nurses Foundation Grant, the UCSF General Clinical Research Center, the UCSF Graduate Student Research Award, and the Wound, Ostomy, and Continence Nursing Scholarship.

\section{Acknowledgement:}

I would like extend my gratitude to Dr. Steven Paul, who provided support in data management and analysis. I also appreciate Dr. Deidre Wipke-Tevis, who helped me to learn the art of charts and figures. My heartfelt thanks to:

- UCSF: Dr. Jergesen \& Dr. Ries, the GCRC, the orthopedic clinic, and 12 Long staff

- El Camino Hospital: the orthopedic physicians, 5E staff \& Admission Unit staff

- All the participants at both hospitals 


\begin{abstract}
Objectives: To examine the effect of external pressure of the bed surface on heel skin temperature in adults in the first three days after hip surgery.
\end{abstract}

Design: A quasi-experimental study in a prospective within subjects repeated-measures design.

Setting: Two acute care hospitals.

Participants: Eighteen subjects (9 men and 9 women) with a mean age of $58.3( \pm 16.1)$ years were recruited after hip surgery at two hospitals.

Methods: Temperature sensors were placed on the plantar surface of each foot, close to the heels. Measures were taken when the heels were 1) suspended above the bed surface for 20 minutes (preload), 2) on the bed surface for 15 minutes (loading), and 3) suspended again above the bed surface for 15 minutes (unloading).

Main Outcome Measures: Heel skin temperature and demographic data.

Results: Heel temperature increased during loading and unloading in both legs on postop days $1(p=0.003)$ and $3(p=0.04)$ but not on post-op day 2 . Heel temperature in the non-operative leg decreased in the first 3 minutes of unloading on post-op days 2 (p = $0.02)$ and $3(\mathrm{p}<0.01)$.

Conclusion: Heel temperature increased with loading and unloading on postoperative days 1 and 3. Upon immediate unloading, hyperemic response was present only in the non-operative leg. Keeping the heels off the bed surface at all times may avoid heel skin temperature changes and prevent tissue damage. Further research is needed to identify the mechanisms that explain the effect of external pressure on heel temperature. 


\section{Introduction}

Pressure ulcers are a frequent complication of hip fracture and its surgical repair. ${ }^{1-}$

${ }^{3}$ The sacrum and heels are the most common sites of pressure ulcer and the incidence of heel ulcers is increasing. ${ }^{4}$ Factors that contribute to heel pressure ulcers in the operative leg include decreased perfusion ${ }^{5}$ and reduction in femoral blood flow during hip surgery. ${ }^{6}$ Likely explanations for decreased perfusion in the non-operative leg are increased pressure at the groin intraoperatively, lateral positioning on the operating table, use of hypotensive anesthesia, and pre-existing vascular diseases. ${ }^{7}$ In addition, the nonoperative leg is often used as a pivot point for turning and repositioning in bed, making it susceptible to intermittent friction and pressure. Consequently, perfusion in both the operative and non-operative legs may be compromised and the heels are at risk of pressure ulcer development. In addition, surgical pain may deter the patient from moving the operative leg, with the heel resting on the bed surface for extended periods of time. Skin temperature has been used as a measure of perfusion, yet the relationship between temperature and perfusion is not linear. ${ }^{8}$ Heat loss in the subcutaneous tissue and the skin is regulated by vasoconstriction and vasodilatation. ${ }^{9}$ Skin temperature is influenced by the difference between ambient temperature and blood temperature and perfusion is correlated with blood temperature. Depending on clinical circumstances, external pressure can increase or reduce skin blood flow. Release of pressure may lead to reactive hyperemia; that is, a transient increase in blood flow. At the other extreme, external pressure can reduce blood flow and produce ischemia, the sine qua non of pressure ulcer development. 
Few studies have explored whether a change in skin temperature is related to external pressure and pressure ulcer development. If a change in skin temperature can reflect skin blood flow, it can also represent the change in external pressure. This study was conducted to examine the relationship between external pressure and heel skin temperature in the non-operative and operative leg over three days after surgery in adults. The study questions were:

1. Is there a difference in heel skin temperature in the non-operative leg compared to the operative leg during repeated loading and unloading conditions in hip surgery patients on postoperative days 1,2 , and 3 ?

2. Is there a difference in heel skin temperature during the first 3 minutes of unloading compared to the last minute of loading on postoperative days 1 , 2, and 3 after hip surgery?

\section{Background}

\section{Blood Flow and External Pressure}

Vascular flow is affected by the application and removal of external pressure. Increased blood flow in the human hand was noted when external pressure of various non-extreme magnitudes was applied. ${ }^{9}$ Pressure-induced vasodilatation is thought to be the body’s protective mechanism against external pressure. In another study, arterial blood flow doubled in the forearm of healthy men when compression stockings were applied to the forearms for 10 minutes. ${ }^{10}$ The increased blood flow and local vessel dilation was attributed to a decrease in transmural pressure in the blood vessel.

\section{Effect of Pressure on Skin Temperature}


Blood flow and external pressure affect skin temperature. A gradual rise in skin temperature at the pressure site was recorded in rats when increasing external pressure was applied. ${ }^{8}$ Similar skin temperature response has been shown in human when trochanter skin was subjected to pressures of $100 \mathrm{mmHg}, 200 \mathrm{mmHg}$, and $300 \mathrm{mmHg}$ for periods of 11 to 67 minutes. With loading, skin temperature increased initially and then decreased but with the removal of pressure, the temperature increased and peaked after 3 to 5 minutes. ${ }^{11}$ The increase in skin temperature during unloading in another study was affected by both the magnitude and the duration of applied pressure. ${ }^{11,12}$ Greater pressure and longer duration resulted in higher post-unloading temperature. A study on the sacrum and gluteus maximus muscle in both older and younger adults also demonstrated that skin temperature increased after removal of external pressure. ${ }^{13}$ Increased perfusion (i.e. reactive hyperemia) was observed when pressure was removed. ${ }^{14}$

\section{$\underline{\text { Skin Temperature \& Pressure Ulcer Formation }}$}

Skin temperature was examined in undamaged but erythematous skin using ultrasonography. ${ }^{15}$ Data showed that capillaries and venules were engorged with red blood cells, suggesting that the engorged blood vessels caused reduced blood flow, persistent erythema, and decreased skin temperature. ${ }^{15}$ Similarly, another study showed that skin perfusion was higher in the center of the nonblanchable erythema (stage 1 pressure ulcer) and decreased toward the edge in patients in the acute care and geriatric units. ${ }^{16}$ It is possible that the increased central temperature reflected inflammation while the decreased distal flow in the small vessels was due to obstruction of vessels with red blood cells. In another study, increased skin perfusion was also found in the damaged area as compared to the undamaged skin on the sacrum in older adults. In this situation, 
the temperature in the damaged skin did not change. ${ }^{17}$ These finding are not consistent with those of others; however, the authors suggest that increased perfusion in the damaged skin might have dissipated enough heat so that skin temperature was not increased.

$\underline{\text { Pain }}$

Pain induces sympathetic nervous activities with subsequent arteriolar vasoconstriction leading to reduced peripheral blood flow ${ }^{18}$ that may impact skin temperature.

\section{Pressure Ulcers \& Hip Surgery Patients}

Pressure ulcers are a frequent complication of hip fracture and surgery ${ }^{3}$ with a common site of ulcer development being the heels. ${ }^{19}$ Perfusion in both legs may be impaired by anesthesia, ${ }^{19}$ operative position, and pre-existing vascular disease. ${ }^{7}$ These unresolved issues led to the present study to explore the relationships between external pressure and temperature of the heels during loading and unloading in postoperative hip fracture patients.

\section{Methods}

In this quasi-experimental study nested within a larger longitudinal study, a prospective within-subjects repeated-measures design was used to study the effects of loading and unloading on skin temperature. An actual clinical condition was used and the weight of each subject's foot served as the loading pressure, with the recognition that loading pressure varies by foot weight and heel architecture. All subjects in this study were positioned on a pressure-reduction mattress (Hill-Rom, Model Advanta) as part of routine nursing care. 
The independent variable was the loading condition with two levels, loading and unloading. The baseline measure (preload), intervention (loading), and post-test measure (unloading) were sequenced as follows: Preload was defined as the time period right before loading and after the 20-minute stabilization when heels were off the bed surface. Studies examining skin temperature on the plantar aspect of the foot found that stabilization of temperature is achieved after 15 minutes. ${ }^{20,21}$ Procedures in this study were consistent with these recommendations, ensuring a stable baseline. Loading was the 15-minute time period when the heels touched the bed (generated by the subject's full weight of the foot); and Unloading was defined as the 15-minute time period when the heels were kept off the bed surface after the heels were placed on the bed surface for 15 minutes. The dependent variable was the heel skin temperature. Subjects were encouraged to maintain maximal pain control during data collection. The non-operative leg served as a comparison to the operative leg.

A convenience sample of 18 subjects was recruited from two San Francisco area hospitals after approval by the Institutional Review Board for each facility as well as that of the University of California, San Francisco. A preliminary power analysis on 9 subjects showed significant differences with $80 \%$ power. Included were persons $\geq 21$ years undergoing hip surgery who had both lower extremities intact, a score $\leq 5$ on the Mental Status Questionnaire (MSQ), an ankle-brachial index (ABI) $\geq 0.9$ in both legs, intact plantar surface foot sensory perception measured with a 5.07 Semmes-Weinstein (S-W) monofilament, and a fasting glucose for diabetics of $<140 \mathrm{mg} / \mathrm{dL}$ or a preoperative random plasma glucose of 120-180 mg/dL. Persons with existing chronic foot ulcers or medical conditions associated with carbon dioxide retention (i.e., chronic 
obstructive airway diseases) were excluded. Eligibility was determined by the researchers through chart reviews, interview of potential subjects and screening tests (MSQ, ABI, and S-W monofilament test). Written informed consent was obtained by the researcher prior to study procedures.

Mental Status Questionnaire (MSQ).

The MSQ objectively measures a person's orientation in time and place, remote memory, and general knowledge. ${ }^{22}$ The number of wrong answers and unanswered items are counted as errors. Cut-points are determined by the number of wrong answers. A score of 6 -10 error points generally indicates moderate to severe mental impairment. ${ }^{23}$ The test-retest reliability is 0.87 at 2 - 4 weeks. $^{24}$ Sensitivity (45\% - 94\%) and specificity (96\% - 99\%) vary according to different cut-points. ${ }^{22}$ We used an error point of $\leq 5$ as an indication that the person was mentally competent to consent and follow the study protocol.

\section{Ankle-brachial Index.}

$\mathrm{ABI}$ is an indirect method of assessing arterial blood flow in the leg. $\mathrm{ABI}$ is obtained using a hand-held Doppler (Park Electronics Model 840) and sphygmomanometer to measure systolic pressures in the brachial, dorsalis pedis and/or posterior tibial arteries. ${ }^{25}$ If arterial blood flow is normal, the pressure in the foot or ankle should be equal or only slightly higher than that in the arm. An index of 1.0 to 1.1 is considered normal, indices below 0.9 indicate the presence of lower extremity arterial disease. ${ }^{25-27}$ Potential subjects with ABI less than 0.9 in either leg were excluded in this study.

Semmes-Weinstein (SW) monofilament test. 
A 5.07 Semmes-Weinstein monofilament ${ }^{28,29}$ was used to assess sensory neuropathy of the feet. The monofilament is applied perpendicular to the skin for about one second with enough force to cause the monofilament to buckle. These monofilaments generate a reproducible bucking pressure and have sizes from 1.65 to 6.65. The higher the number of the monofilament, the more difficult it is to bend, and a greater force must be applied to bend the monofilament for the patient to feel the pressure. $^{30}$ The inability to feel a point of pressure over the metatarsal heads and toes, the medial or lateral midfoot on the plantar surface of the foot (as in diabetic neuropathy) represents a sensory loss of about $98 \%{ }^{28}$ We required subjects to have intact plantar sensation, since neuropathy may alter temperature and blood flow responses, and increase the risk of pressure ulcers.

Measurements and Instruments

Demographic data.

Demographic data extracted from the participants' medical record or obtained by interview were age, gender, ethnicity and race, smoking history, blood glucose level, comorbid medical conditions, type of anesthesia, duration of surgery, estimated blood loss during surgery, mobility, pain medications and sedatives.

\section{Heel skin temperature.}

Heel skin temperature was measured with a Respiratory Support Products (RSP) Bi-Temp temperature monitor (model TM-201) (Smiths Industries Medical Systems, London) using 400 series thermistors in contact with the skin surface. Sensors covered by tape produced higher mean skin temperatures than uncovered sensors $(\mathrm{p}<0.05) .^{31}$ To mitigate this error, self-adherent sensors were utilized (i.e., no tape was used to hold 
sensors in place). All measurements were conducted in the patient's hospital room and room temperature during data collection was $21-23^{\circ} \mathrm{C}$, with a humidity of $60 \%$.

Pain.

Pain was assessed using a horizontal Visual Analog Scale (VAS) and when present, location was identified on a picture of a body outline. The VAS measured pain intensity on a $10 \mathrm{~cm}$ scale $(0 \mathrm{~cm}$ = no pain, $10 \mathrm{~cm}$ = worst pain you can imagine $)$ where the participant marked the line at the point that best reflected their pain intensity "at that particular moment" ${ }^{32}$ The VAS is a reliable measure of subjective pain in the clinical setting. ${ }^{32-35}$

Procedure

Data were collected on both legs on the first three post-operative days. To simulate clinical practice, measures were performed with subjects in their natural clinical condition. Some subjects wore anti-embolic stockings (TED Hose, Kendall, Mansfield) on all 3 days while some wore them intermittently. For those with anti-embolic stockings, the stocking (plantar foot portion) was pulled back through the opening at the posterior aspect of the toes and the thermister attached to the plantar surface of the foot adjacent to the heel. The sensors did not touch the bed surface when the heels were placed on the bed. For participants with the intermittent pneumatic compression devices (IPC) on, the device was not manipulated as it extended only to the ankle. The thermister was applied to the plantar surface of the foot in a similar location on all subjects.

All participants were positioned supine in bed. A pillow was placed under each calf to suspend the heels at about 15 degrees from the bed surface. The legs were covered 
by a single bed sheet and maintained for 20 minutes. At the end of 20 minutes, baseline values of pain intensity (and location, if applicable) and skin temperature were recorded. Loading was performed by removing the pillows and placing the heels in contact with the bed surface. Heel skin temperature was recorded every 3 minutes for 15 minutes and at the end of loading. Both skin temperature and pain were assessed and recorded at the end of loading.

Then, the heels were again suspended above the bed surface on pillows. During this 15 minutes of unloading, heel skin temperature was measured every minute for the first 3 minutes and then every three minutes for the remaining 12 minutes. At the end of unloading, both skin temperature and pain were assessed and recorded. Subjects were encouraged to use patient-controlled-analgesia (PCA) and oral analgesic for maximum pain control before and during data collection. All data were collected by the researchers.

\section{Data Analysis}

The Statistical Package for the Social Sciences (Version 13.0) was used to manage and analyze data. All variables were examined descriptively. Data were analyzed with a 3-way repeated measures analysis of variance (RMANOVA) with 3 within subjects' factors: leg, day, and time. The leg had two levels: operative leg and non-operative leg. Day had three levels: post-operative day 1, post-operative day 2, and post-operative day 3. Time had 11 levels: preload, loading (3, 6, 9, 12, and 15 minutes), and unloading (1, 2, 3, 6, 9, 12, and 15 minutes). The 3-way RMANOVA design tested the main effect of day, leg, and pressure loading time as well as the interaction of day $\mathrm{x}$ leg, day x pressure loading time, leg x pressure loading time, and day x leg x pressure loading time. Prospectively, a follow-up test for contrast was planned, and subsequently 
used, when any of the main effects was significant; similarly, for any interaction, tests of simple effects or tests of trends were planned and performed. A conservative value for these post-hoc tests was set prospectively at 0.05 with significance for any one contrast equal to 0.05 divided by the total number of contrasts. This meant that an alpha of $<0.02$ was the criterion for 3 contrasts, $<0.01$ was the criterion for 5 contrasts, and $<0.005$ was the criterion for 10 contrasts. The relationship of pain and skin temperature was evaluated using Pearson Product Moment Correlation. In addition, the relationships between demographic data and the dependent variable were examined using correlation analysis.

\section{Results}

\section{Sample}

Forty people were approached for inclusion in the study and 18 participated. Of the 22 who were not enrolled, 15 refused, 6 had their surgery cancelled or postponed, and one did not meet the screening criteria. Nine subjects were recruited from each of the two sites.

The mean age of the sample was $58.3 \pm 16.1$ years. Half of the subjects were women, 61.1\% were Caucasian, 22.2\% were Asian, 11.1\% African American, and 5.6\% Hispanic. Most had degenerative joint disease (88.9\%), while a small percentage had a fracture (11.1\%). All subjects had one or more concurrent diseases, with hypertension being the most frequent secondary diagnosis (27.8\%), followed by coronary artery disease (11.1\%). Mean ABI in 13 subjects was $1.0 \pm 0.08$ in the non-operative leg and $1.0 \pm 0.14$ in the operative leg, indicating peripheral arterial disease was unlikely. Five subjects refused ABI measurement but had no documented peripheral arterial disease. 
One subject was a current smoker. No subjects had sensory loss in their feet or had diabetes mellitus. Most persons underwent general anesthesia (83.3\%), were American Society of Anesthesia (ASA) classification II (58.5\%), with a median surgery duration of 2.5 hours (interquartile range 111 - 285 minutes), and median estimated blood loss of 400 $\mathrm{ml}$ (interquartile range 200 - $650 \mathrm{ml}$ )(Table 1). Pain assessment during preload, loading, and unloading yielded a range of scores from 0 to 8 . Most subjects $(n=14)$ denied having pain or had minimal pain while resting in bed. A few subjects $(n=4)$ complained of moderate to severe pain due to muscle spasm. Pain level did not fluctuate during the 2 hours of data collection.

Study Question 1: Was there a difference in heel skin temperature in the non-operative leg compared to the operative leg during repeated loading and unloading conditions in hip surgery patients on postoperative day 1,2 , and 3 ?

There was an overall trend of change in heel skin temperature in both legs during loading and unloading compared to preload $(\mathrm{p}<0.01)$ on all 3 post-op days (Figure 1 ). Heel skin temperature in both legs increased during loading and unloading on post-op days $1(\mathrm{p}<0.01)$ and $3(\mathrm{p}=0.04)$. Heel skin temperature on post-op day 2 was not significantly different and there was no significant difference in the skin temperature in the operative and non-operative leg. Mean heel skin temperature on each leg over the first 3 post-operative days is presented in Table 2 .

Study Question 2: Was there a difference in heel skin temperature during the first 3 minutes of unloading compared to the last minute of loading on post-operative day 1,2 , and 3 after hip surgery? 
Heel skin temperature in the non-operative leg decreased in each of the first 3 minutes of unloading only on post-op days $2(\mathrm{p}=0.02)$ and $3(\mathrm{p}<0.01)$. There was no heel skin temperature change in the first 3 minutes in the operative leg over the 3 days $(\mathrm{p}>0.05)$ (Figure 2).

\section{Discussion}

This study examined the relationships between external pressure and heel skin temperature in the operative and non-operative leg after hip surgery in 18 adults. Heel skin temperature increased during loading and unloading on days 1 and 3 in both legs. Heel skin temperature decreased only in the non-operative leg during the first 3 minutes of unloading on post-op days 2 and 3. Since the difference in skin temperature was small and relatively inconsistent when comparing the three post-op days, the data must be interpreted with caution. There was a possibility that the statistical difference detected was secondary to a potential lack of clinical significance in the difference in skin temperature.

\section{Loading}

Heel skin temperature in our study increased significantly during loading. This finding is consistent with the findings of Sae-Sia and colleagues (2007) who demonstrated an increase in sacral skin temperature during pressure loading of 2 hours. ${ }^{36}$ The mean sacral skin temperature at the end of the 2-hour lying in the supine position (loading) was compared to that in the lateral position (baseline) in subjects with spinal cord injury $(n=20)$, orthopedic trauma $(n=30)$, and healthy conditions $(n=47) .{ }^{36}$ The increase in sacral skin temperature during loading was observed in all study groups but was the lowest in the spinal cord injury group when compared with the other 2 groups (p 
$<0.01)$. The authors suggested that people with spinal cord injury may have problems with microvascular function, contributing to the difference in temperature response. Other studies confirm that loading increases skin temperature but the variability in duration of loading may confound these temperature responses. ${ }^{8,11}$

Increase in skin temperature has been shown in pressure induced erythema in the ischial tuberosity in 6 individuals with spinal cord injury at or above the thoracic level. Skin temperature at the erythematous center remained elevated one hour after pressure relief. ${ }^{37}$ The authors postulated that the elevated skin temperature was due to an increased capillary flow in the underlying tissue, increased tissue metabolism, and a mild inflammatory response. Another study on hospitalized neurologically impaired patients in Thailand revealed that increased sacral skin temperature predicted subsequent pressure ulcer development. ${ }^{38}$ Mean sacral skin temperature in subjects who developed a pressure ulcer (stage 1 and II) was higher than those who did not develop a pressure ulcer during both loading $(\mathrm{p}<0.01)$ and unloading $(\mathrm{p}<0.01)$ at both the initial and subsequent assessments. These findings may be confounded by the fact that this study was conducted in a non-air conditioned hospital in hot temperatures and patients may have experienced changes in their thermoregulatory mechanism from neurological damage. ${ }^{38}$ In addition, these subjects were either bed- or chair-bound before data collection and deep tissue damage might have already occurred. Regardless, the increased sacral skin temperature during loading and unloading was associated with compromised tissue perfusion and pressure ulcer development.

The relationship among inflammation, blood flow, and skin temperature was examined in a study that compared skin blood flow, skin temperature, and transcutaneous 
oxygen on the medial aspect of the lower leg in subjects with $(n=31)$ and without chronic venous disease $(n=24) .{ }^{39}$ Measurements were taken once a week for three consecutive weeks with laser Doppler, infrared thermometer, and transcutaneous oximeter respectively. Participants with chronic venous disease had no open ulcers. Skin temperature was found to be consistently higher at weeks 1 , 2, and 3 ( $p<0.05$ ), blood flow was higher at weeks 1 and 3, while transcutaneous oxygen was lower at weeks 1 and 3 in the chronic venous disease group. ${ }^{39}$ The authors suggested that inflammation might have affected heat production and retention in the skin. However, no direct measure of inflammation was reported in this study.

Loading in our study produced increased local skin temperature on the heels, a possible indication of tissue inflammation under the skin. However, with only 15 minutes of loading, it is unlikely that inflammation was the major cause. Since increased skin temperature has been shown to involve deep tissue, ${ }^{40}$ leaving the heels on the bed surface may have further increased skin temperature, leading to a higher risk of heel tissue damage. The mattress and its covers also may retain heat, producing a warming effect on the heels.

The reduction in skin blood flow in our study in response to external pressure is inconsistent with other studies that have shown increased skin blood flow with various loading magnitudes. ${ }^{9-10}$ Of interest are the variations in blood flow in response to the magnitude of pressure in a study on 10 young adults. Incremental indentation was applied to the sacrum using a computer-controlled indenter; skin blood flow was measured by laser Doppler. ${ }^{41}$ The indenter system consisted of a $36 \mathrm{~mm}$ diameter probe which incorporated the indenter, temperature sensor, heating head, and laser Doppler 
probe. Mean skin blood flow decreased as pressure increased from 0 to $15 \mathrm{mmHg}$ but increased as pressure increased from 15 to $60 \mathrm{mmHg}$. The authors suggested a myogenic mechanism may be responsible for these differences where skin blood flow is mediated by changes in transmural pressure of the blood vessels. ${ }^{41}$ Loading pressure in our study was not controlled or measured but the variations in transmural pressure may have contributed to changes in skin blood flow.

Tissue damage, inflammation and the warmth created from wearing external stockings might also partially explain the increased skin temperature. Heat can be trapped by stockings, sequential compression devices, and cover sheets. Since skin blood flow plays an important role in transporting heat from the skin surface, external pressure that impedes blood flow may lead to an increase in skin temperature. ${ }^{42}$ Perfusion in our study decreased during loading as reflected in the reduction of skin oxygenation. ${ }^{43}$ The corresponding increase in skin temperature may be related to impaired heat dissipation instead of increased perfusion.

\section{Unloading}

An increase in skin temperature during the immediate unloading period is usually indicative of reactive hyperemia. This study sought to evaluate reactive hyperemia by examining temperature minute by minute for the first 3 minutes of unloading. During the immediate pressure unloading period, a decrease in heel skin temperature was evident in the non-operative leg on post-op days 2 and 3. The operative leg did not show a reduced temperature response, thus, heat dissipation and perfusion might be different between the non-operative and operative legs. Other investigators have also reported a reduction in skin temperature during the immediate unloading period before temperature increased 
again. ${ }^{36}$ Thermography of the forearm after loading showed a 1-3 minute time lapse before temperature increased to a maximum. ${ }^{44}$ Differences may be due to blood flow as upper extremities consistently have better perfusion than heels. In another study, the immediate decrease in skin temperature during unloading was shown when the thermal response of cyclic pressure on the lower leg of human subjects $(n=3)$ was examined. ${ }^{45}$ Skin temperature was recorded every 30 seconds in this small sample at the pressure unloading site and a contra lateral site using an infrared temperature sensor. Temperature decreased immediately after unloading and then increased, prompting authors to postulate reperfusion as a possible mechanism. In our study, skin temperature was recorded every 60 seconds for the first three minutes, and therefore may not have captured a very brief reduction in temperature at the beginning of unloading. Whether the sudden decrease in temperature represents an increase in blood flow after release of pressure in the nonoperative leg is not clear.

After the brief period of hyperemia (the immediate first three minutes of unloading), heel skin temperature during the remaining unloading period increased in both legs on postoperative days 1 and 3. It is unclear why there was no significant change in skin temperature during unloading on day 2. Other studies have also shown a rise in skin temperature during unloading. ${ }^{11,13,14}$ Skin temperature in these studies remained elevated even in the immediate unloading period.

Pain

Literature on the relationship between pain and skin temperature is scarce. For example, it is not known if pain relates to a higher skin temperature in the peri-wound area or if changes in skin temperature indicate perfusion changes. ${ }^{38}$ Pain could be both a 
confounder and an effect modifier. Further work is needed to understand the role of pain and temperature.

\section{Strengths and Limitations}

Heel skin temperature was measured in the clinical environment. Although the environmental temperature and humidity were fairly constant, there was some variation in heel skin temperature within individuals over the 3 days. The differences were small and are consistent with normal subject's core body temperature variability. Temperature variance was greater on post-op days 2 and day 3 than on day 1 . Further research needs to systematically control or measure factors that might contribute to this phenomenon, such as sequential compression devices, leg mobility, and edema. Most subjects were wearing anti-embolic stockings during data collection and this may have had a warming effect on the feet, confounding the interpretation of the findings. Due to the small sample size of subjects wearing leg devices, it was not meaningful to examine the variance separately. Because of the technical difficulty of placing the sensors, consistent sensor placement could not be assured on each data collection day, and this may have contributed to intrasubject variability. Pain after hip surgery could be a confounder or effect modifier. Since subjects were encouraged to use pain medications, there is no variability in the data to examine pain as a variable. Despite these limitations, this study demonstrates a heel skin temperature response to external pressure in the clinical setting.

\section{Implications and Recommendations}

The important finding from this study is that there was a trend for heel skin temperature to increase after placing the heels on the bed surface for 15 minutes and this increase continued after the heels were removed from the bed surface. Even this 
relatively short period of pressure loading seemed to have a lasting effect on skin temperature. Keeping the heels off the bed surface at all times may avoid heel skin temperature changes, enhance heat dissipation, and prevent deep tissue damage. The inconsistent temperature change in the operative leg upon unloading suggests possible inflammation, edema, impaired perfusion or heat dissipation. This finding underscores the fact that post-operative patients are at significant risk for pressure ulcer development and pressure ulcer prevention is imperative after hip surgery.

Future studies on the development of heel pressure ulcers need to explore the response of heel skin temperature to bilateral pressure loading and unloading longitudinally in an age and gender matched non-surgical population. Equally important is the examination of the effect of edema and compression stockings on heel skin temperature, and whether skin temperature is an accurate indicator of pressure-induced reactive hyperemia. Finally, studies must examine whether ethnicity impacts skin temperature. 


\section{References}

1. Lichtblau S. Hip fracture. Surgical decisions that affect medical management. Geriatrics 2000; 55(4): 50-52, 55-56.

2. Margolis DJ, et al. Medical conditions as risk factors for pressure ulcers in an outpatient setting. Age Ageing 2003; 32(3): 259-64.

3. Soderqvist A, Ponzer A, Tidermark J. Cognitive function and pressure ulcers in hip fracture patients. Scand J Caring Sci 2007; 21(1): 79-83.

4. Cuddigan J, Berlowitz DR, Ayello EA. Pressure ulcers in America: Prevalence, incidence, and implications for the future. Advances in Skin and Wound Care 2001; 14(4): 208-215.

5. Martin JT. Positioning aged patients. Anesthesiology Clinics of North America 2000; 18(1): 105-121.

6. Hupel TM., et al. Blood flow changes to the proximal femur during total hip arthroplasty. Can J Surg 2000; 43(5): 359-364.

7. Smith JW, et al. Complications after total hip replacement. The contralateral limb. J Bone Joint Surg Am 1989; 71(4): 528-535.

8. Herrman EC, et al. Skin perfusion responses to surface pressure-induced ischemia: Implications for the developing pressure ulcer. Journal of Rehabilitation Research \& Development 1999; 26(2): 109-120.

9. Abraham P, et al. Dynamics of local pressure-induced cutaneous vasodilation in the human hand. Microvasc Res 2001; 61(1): 122-9.

10. Bochmann RP, et al. External compression increases forearm perfusion. J Appl Physiol 2005; 99(6): 2337-44. 
11. Mahanty SD, Roemer RB. Thermal response of skin to application of localized pressure. Arch Phys Med Rehabil 1979; 60(12): 584-90.

12. Mahanty SD, Roemer RB, Meisel H. Thermal response of paraplegic skin to the application of localized pressure. Arch Phys Med Rehabil 1981; 62(12): 608-11.

13. Schubert V, Fagrell B. Evaluation of the dynamic cutaneous post-ischaemic hyperaemia and thermal response in elderly subjects and in an area at risk for pressure sores. Clinical Physiology 1991; 11(2):169-182.

14. Sprigle S, et al. Clinical skin temperature measurement to predict incipient pressure ulcer. Advances in Skin and Wound Care 2001; 14(3): 133-137.

15. Sato M, et al. Prognosis of stage I pressure ulcers and related factors. Int Wound J 2006; 3(4): 355-62.

16. Lindgren $\mathrm{M}$, et al. Altered skin blood perfusion in areas with non blanchable erythema: an explorative study. Int Wound J 2006; 3(3): 215-23.

17. Schubert V, Perbeck L, Schubert P A. Skin microcirculatory and thermal changes in elderly subjects with early stage of pressure sores. Clinical Physiology 1994; 14(1): 1-13.

18. Guyton AC, Hall JE. Textbook of Medical Physiology. 10th ed. Philadelphia: W. B. Saunders Company, 2000.

19. Edwards JL, Pandit H, Popat MT. Perioperative analgesia: A factor in the development of heel pressure ulcers. British Journal of Nursing 2006; 15(6): S205.

20. Sun PC, Jao SH, Cheng CK. Assessing foot temperature using infrared thermography. Foot Ankle Int 2005; 26(10): 847-53. 
21. Sun PC, et al. Relationship of skin temperature to sympathetic dysfunction in diabetic at-risk feet. Diabetes Res Clin Pract 2006; 73(1): 41-6.

22. McDowell I, Newell C. Measuring Health. A Guide to Rating Scales \& Questionnaires. 2nd ed. New York: Oxford University Press, 1996.

23. Zarit SH, Miller NE, Kahn RL. Brain function, intellectual impairment and education in the aged. J Am Geriatr Soc 1978; 26(2): 58-67.

24. Lesher EL, Whelihan WM. Reliability of mental status instruments administered to nursing home residents. J Consult Clin Psychol 1986; 54(5):726-7.

25. Bonham PA, and Flemister BG. Guideline for management of wounds in patients with lower-extremity arterial disease, Wound Ostomy and Continence Nurses Society: IL. p. 1-33, 2002.

26. Whiteley MS, Fox AD, Horrocks M. Photoplethysmography can replace handheld Doppler in the measurement of ankle/brachial indices. Annals of the Royal College of Surgeons of England 1998; 80(2): 96-98.

27. Zink M, Rousseau P, Holloway Jr GA. Lower Extremity Ulcers, in Acute and Chronic Wounds, R.A. Bryant, Editor. Mosby Year Book: St. Louis. p. 164-212, 1992.

28. Jeng C, Michelson J, Mizel M. Sensory thresholds of normal human feet. Foot Ankle Int 2000; 21(6): 501-4.

29. Barber MA, et al. Evaluation of pressure threshold prior to foot ulceration: oneversus two-point static touch. J Am Podiatr Med Assoc 2001; 91(10): 508-14.

30. Zangaro GA, Hull MM. Diabetic neuropathy: Pathophysiology and prevention of foot ulcers. Clinical Nurse Specialist 1999; 13(2): 57-65. 
31. Buono MJ, Ulrich RL. Comparison of mean skin temperature using 'covered' versus 'uncovered' contact thermistors. Physiol Meas 1998; 19(2): 297-300.

32. McGuire DB. Measuring Pain, in Instruments for clinical health-care research, Stromborg MP, Olsen SJ, Editor, Jones \& Bartlett Publishers: Boston. p. 528-544, 1997.

33. Bergh I, et al. An application of pain rating scales in geriatric patients. Aging (Milano) 2000; 12(5): 380-387.

34. McCormack H, Horne D, Sheather S. Clinical applications of visual analog scales: a critical review. Psychological Medicine 1988; 18:1007-1019.

35. Salo D, et al. Can patients accurately read a visual analog pain scale? Am J Emerg Med 2003; 21(7): 515-9.

36. Sae-Sia W, Wipke-Tevis DD, Williams DA. The effect of clinically relevant pressure duration on sacral skin blood flow and temperature in patients after acute spinal cord injury. Arch Phys Med Rehabil 2007; 88(12):1673-80.

37. Finestone HM, et al. Erythema and skin temperature following continuous sitting in spinal cord injured individuals. J Rehabil Res Dev 1991; 28(4): 27-32.

38. Sae-Sia W, Wipke-Tevis DD, Williams DA. Elevated sacral skin temperature (Ts): A risk factor for pressure ulcer development in hospitalized neurologically impaired Thai patients. Appl Nurs Res 2005; 18(1): 29-35.

39. Kelechi TJ, Michel Y. A descriptive study of skin temperature, tissue perfusion, and tissue oxygen in patients with chronic venous disease. Biol Res Nurs 2007; 9(1): 70-80. 
40. Kokate JY, et al. Temperature-modulated pressure ulcers: a porcine model. Arch Phys Med Rehabil 1995; 76(7): 666-73.

41. Brienza DM, Geyer MJ, Jan YK. A comparison of changes in rhythms of sacral skin blood flow in response to heating and indentation. Archives in Physical \& Medical Rehabilitation 2005; 86(G): 1245-1251.

42. Jaszczak P. Blood flow rate, temperature, oxygen tension and consumption in the skin of adults measured by a heated microcathode oxygen electrode. Dan Med Bull 1988; 35(4):322-34.

43. Wong VK, et al. How heel oxygenation changes under pressure. Wound Repair Regen 2007; 15(6): 786-94.

44. Goller $\mathrm{H}$, et al. The effect of external pressure on skin temperature distribution by thermography. Med Res Eng 1976; 12(1): 6-8.

45. Sanders JE. Thermal response of skin to cyclic pressure and pressure with shear: a technical note. J Rehabil Res Dev 2000; 37(5): 511-5. 
Table 1.

Surgery Related Information

\section{Percentage}

Previous hip surgery

Type of anesthesia

General

Epidural/spinal

ASA classification (physical status)

I

II

III

IV
44.4

83.3

15

16.7

5.6

$55.5 \quad 10$

33.3

5.6

1
Median

Median duration of surgery in minutes (IQR) 160 (174)

Median estimated blood loss in millimeter (IQR) 400 (450)
8

3

1

6

$\mathbf{n}$

8

5

(1)

\begin{tabular}{lcc} 
& Median & $\mathbf{n}$ \\
\hline Median duration of surgery in minutes (IQR) & $160(174)$ & 18 \\
Median estimated blood loss in millimeter (IQR) & $400(450)$ & 18 \\
\hline
\end{tabular}


Table 2.

Mean Heel Skin Temperature in the Non-operative and Operative Leg $(n=18)$

Conditions

Mean heel temp $\left({ }^{\circ} \mathrm{C}\right)(\mathrm{SD})$

Non-operative leg Operative leg

Post-op day 1

Preload

32.6 (2.3)

32.9 (2.7)

33.1 (2.7)

33.1 (2.5)

Loading (15 mins)

$33.3(2.5)$

$33.5(2.4)$

Post-op day 2

Preload

33.0 (3.3)

32.7 (3.4)

33.1 (2.6)

33.2 (3.3)

33.3 (2.5)

33.1 (3.2)

Unloading (15 mins)

32.0 (3.4)

32.1 (3.3)

$33.1(3.4)$

33.1 (3.3)

$33.3(3.2)$

$33.4(3.2)$

Post-op day 3

Preload

Unloading (15 mins) 
Figure 1.

Mean heel skin temperature ( \pm SD) during preload, loading, and unloading in the operative and non-operative leg on the first 3 post-operative days.

Heel skin temperature in both legs increased over time during loading and unloading as compared to preload $(p=$ 0.001). The change over time did not depend on the day or the leg.

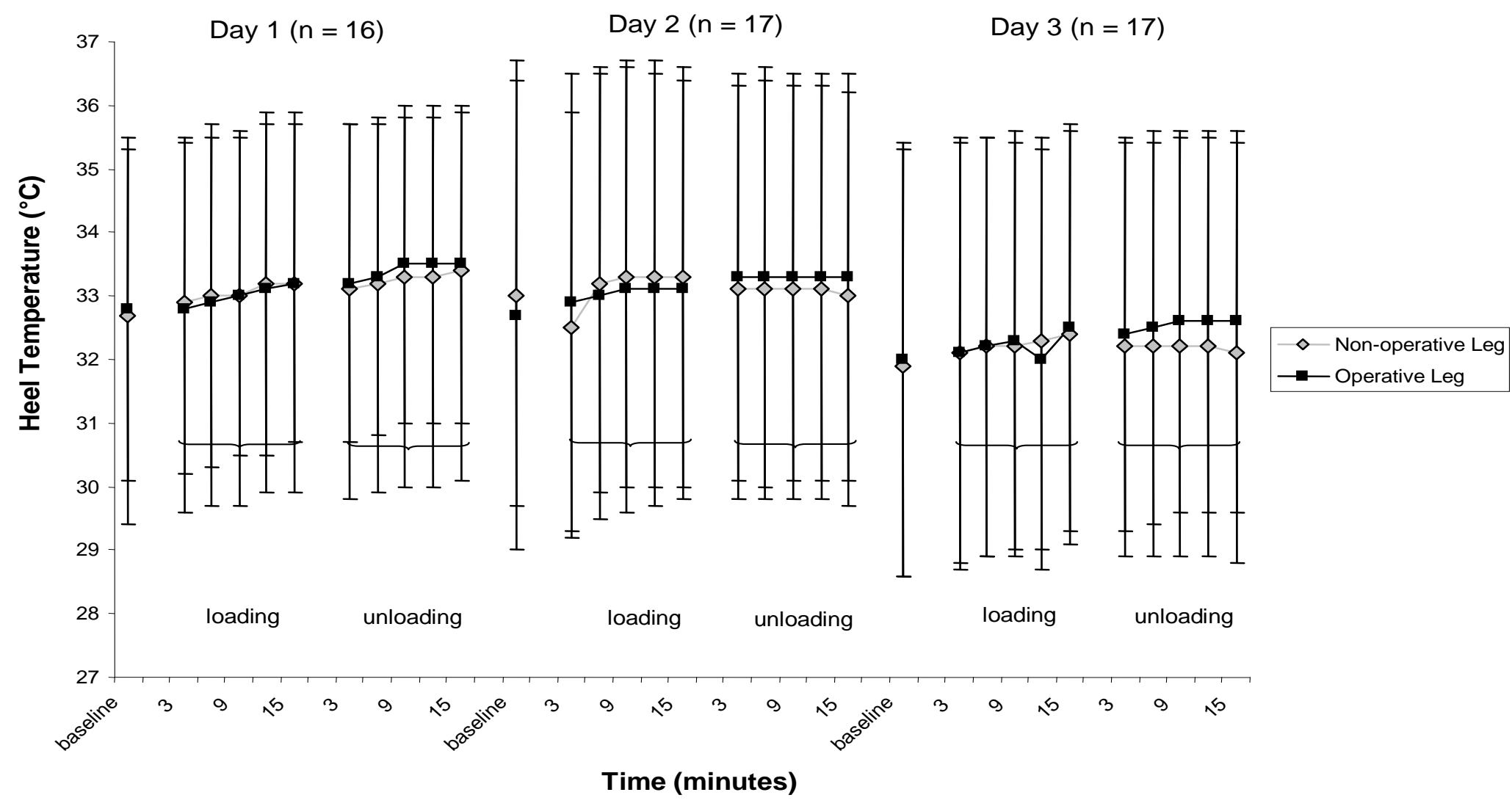




\section{Figure 2.}

Mean heel skin temperature $( \pm S D)$ in the non-operative leg and operative leg during the first 3 minutes of unloading on the first 3 post-operative days.

Heel skin temperature in the non-operative leg did not change from the 15th minute of loading to the first 3 minutes of unloading on post-op day 1 . Heel skin temperature decreased in the first 3 minutes of unloading on post-op days $2(p=0.021)$ and $3(p=0.002)$. Heel skin temperature in the operative leg did not differ in the first 3 minutes of unloading from loading at the 15th minute in all 3 post-operative days.

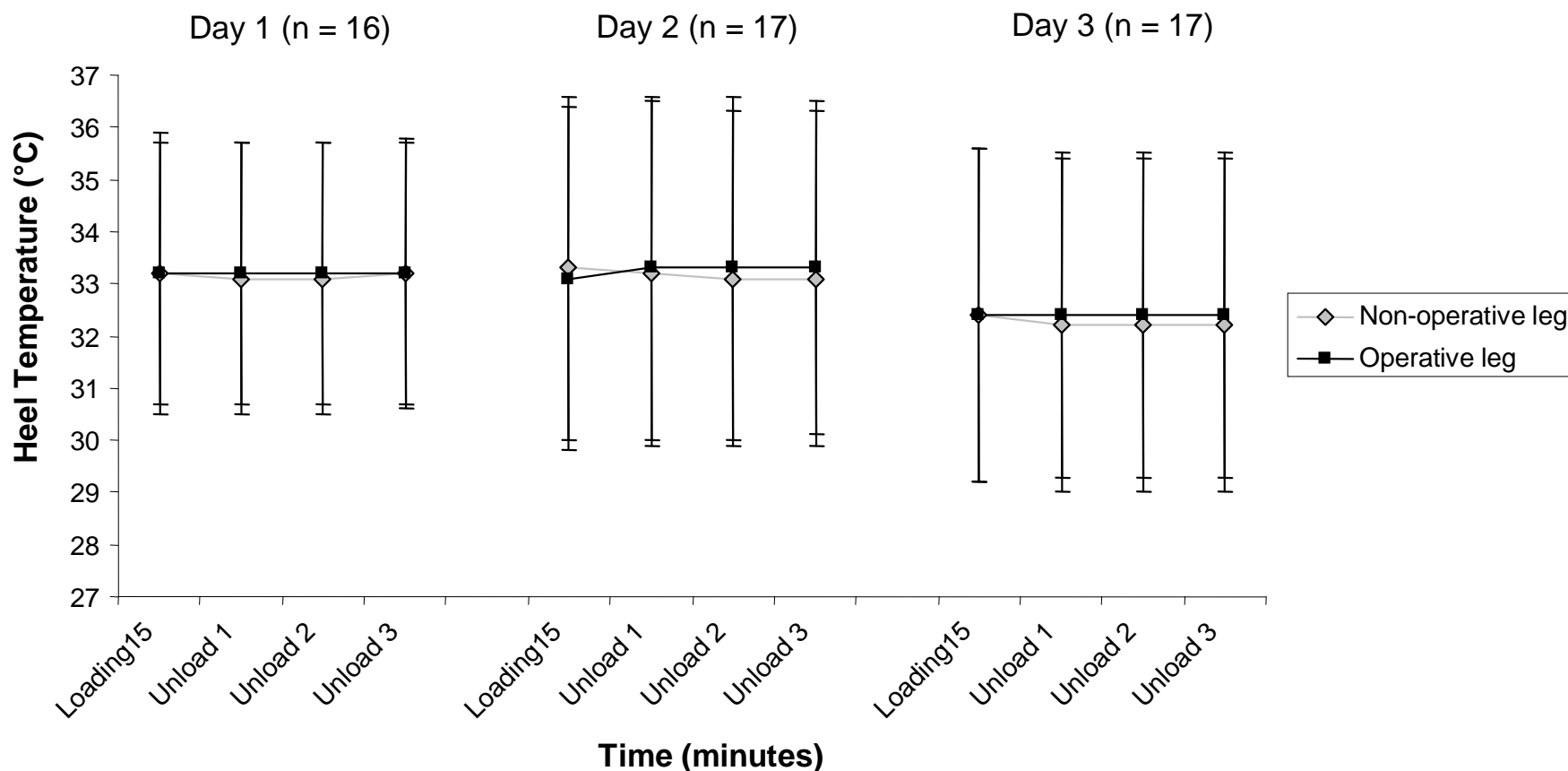

